\title{
Roles of programmed death protein 1/ programmed death-ligand 1 in secondary brain injury after intracerebral hemorrhage in rats: selective modulation of microglia polarization to anti-inflammatory phenotype
}

\author{
Jie Wu${ }^{\dagger}$, Liang Sun ${ }^{\dagger}$, Haiying Li, Haitao Shen, Weiwei Zhai, Zhengquan Yu ${ }^{*}$ and Gang Chen ${ }^{*}$
}

\begin{abstract}
Background: Microglia and its polarization play critical roles in intracerebral hemorrhage-induced secondary brain injury. Programmed death protein 1/programmed death-ligand 1 has been reported to regulate neuroimmune cell functions. Signal transducers and activators of transcription 1 participate in microglia polarization, and programmed death protein 1/programmed death-ligand 1 could regulate the activation of signal transducers and activators of transcription 1. We herein show the critical role of programmed death protein 1/programmed death-ligand 1 in the polarization of microglia during intracerebral hemorrhage-induced secondary brain injury in rat models.

Methods: An autologous blood intracerebral hemorrhage model was established in Sprague Dawley rats (weighing 250-300 g), and primary cultured microglia was exposed to oxyhemoglobin to mimic intracerebral hemorrhage in vitro. Specific siRNAs and pDNA for programmed death protein 1 and programmed death-ligand 1 were exploited both in vivo and in vitro.

Results: In the brain tissue around hematoma, the protein levels of programmed death protein 1 and programmed death-ligand 1 and the interaction between them, as well as the phosphorylation of signal transducers and activators of transcription 1, were higher than that of the sham group and collectively peaked at $24 \mathrm{~h}$ after intracerebral hemorrhage. Overexpression of programmed death protein 1 and programmed death-ligand 1 ameliorated intracerebral hemorrhage-induced secondary brain injury, including brain cell death, neuronal degeneration, and inflammation, while their knockdown induced an opposite effect. In addition, overexpression of programmed death protein 1 and programmed death-ligand 1 selectively promoted microglia polarization to anti-inflammation phenotype after intracerebral hemorrhage and inhibited the phosphorylation of signal transducers and activators of transcription 1, suggesting that intracerebral hemorrhage-induced increases in programmed death protein 1 and programmed death-ligand 1 maybe a self-help.
\end{abstract}

Conclusions: Enhancing the expressions of programmed death protein 1 and programmed death-ligand 1 may induce a selective modulation of microglia polarization to anti-inflammation phenotype for intracerebral hemorrhage treatment.

Keywords: Intracerebral hemorrhage, Secondary brain injury, Programmed death protein 1, Programmed death-ligand 1, Microglia polarization

\footnotetext{
*Correspondence: 13280008348@163.com; nju_neurosurgery@163.com Jie Wu and Liang Sun are co-first authors.

Gang Chen and Zhengquan Yu are co-corresponding authors.

${ }^{\dagger}$ Equal contributors

Department of Neurosurgery \& Brain and Nerve Research Laboratory, The First Affiliated Hospital of Soochow University, 188 Shizi Street, Suzhou, Jiangsu Province 215006, China
} 


\section{Background}

Intracerebral hemorrhage ( $\mathrm{ICH})$ accounts for $\sim 15 \%$ of stroke cases in developed countries and $\sim 50 \%$ or more in developing countries, especially in regions of Asia with high incidence of hypertension [1-3]. And ICH severely reduces the quality of life of patients, with a 5-year survival rate of $\sim 30 \%[4,5]$. Beside blood clot-induced primary injury in the early phase [6], a series of chemical and immune responses known as secondary brain injury (SBI) seriously affect the prognosis of patients and is likely to be subjected to intervention [7-9].

Recent reports have shown that microglia/macrophages are the main regulatory cells in the immune defense response of the central nervous system (CNS) that affect subsequent inflammatory processes $[10,11]$. After brain injury, a large number of microglia and macrophages around the hematoma rapidly respond and release effector molecules $[12,13]$. Numerous recent studies have shown that in response to brain injury, different phenotypes of microglia/macrophages are observed and play various roles based on signals released in the microenvironment $[11,13]$. Two main phenotypes of microglia/macrophages are pro-inflammatory phenotype and antiinflammatory phenotype. Pro-inflammatory phenotype promotes inflammation and kills microorganisms, while anti-inflammatory inhibits inflammation and is involved in tissue repair and reconstruction [13]. Microglia activation and polarization during experimental intracerebral hemorrhage has been reported [14, 15].

Programmed death-1 (PD-1; also known as CD279 or PDCD1), which is a transmembrane glycoprotein of the CD28/cytotoxic $\mathrm{T}$ lymphocytes associated antigen-4 (CTLA-4) immunoglobulin superfamily, was first discovered in 1992 [16]. Its ligands, programmed death-ligand 1 (PD-L1) (B7-H1) and PD-L2 (B7-CD), are type I transmembrane proteins of the $\mathrm{B} 7$ family. PD-1 inhibits phosphatidylinositol-3-kinase (PI3K) and protein kinase B (Akt) activities [17]. The PI3K-Akt signaling pathway is involved in mediating phosphorylation of signal transducers and activators of transcription 1 (STAT1) [18], which has been reported to promote pro-inflammatory phenotype polarization of microglia [19]. PD-L2, another ligand of PD-1, was reported to have a threefold higher affinity for PD-1 compared with PD-L1 [20]. However, studies of the PD-1/PD-L2 signaling pathway have been rarely reported. Numerous studies have shown that PD$1 /$ PD-Ls play important roles in mediating CNS disorders such as ischemic stroke [21], multiple sclerosis [22], and Alzheimer's disease [23].

In conclusion, accumulating researches suggested a therapeutic potential of PD-1/PD-Ls in ICH-induced SBI via regulating microglia polarization. However, until now, no study has investigated the contribution of PD1/PD-Ls to microglia polarization. Therefore, the aim of this study was to investigate the role of PD-1/PD-Ls and to assess the therapeutic potential of PD-1/PD-Ls in $\mathrm{ICH}$-induced SBI.

\section{Methods}

Experimental animals

Adult male Sprague Dawley rats weighing 250-300 g were purchased from the Laboratory Animal Center, Medical College of Soochow University, Suzhou, Jiangsu, China. The animal experimental protocols were approved by the Animal Care and Use Committee of Soochow University and complied with the ARRIVE guidelines. All animals were housed in a quiet environment maintained at $18-22{ }^{\circ} \mathrm{C}$ with stable humidity, and animals had free access to food and water.

\section{$\mathrm{ICH}$ modeling in rats}

As described by Deinsberger et al., ICH modeling in rats was performed by injecting autologous arterial blood into the basal ganglia [24]. Rats were anesthetized by intraperitoneal injection of $10 \%$ chloral hydrate $(36 \mathrm{mg} /$ $100 \mathrm{~g}$ bodyweight). After successful anesthesia, rats were placed in the prone position, and their heads were fixed with a stereotaxic frame (ZH-Lanxing B-type stereotaxic frame, Anhui Zhenghua Biological Equipment Co., Ltd. Anhui, China). The drill site of each animal was determined according to the stereotaxic atlas of the rat brain (arterial blood injection in this experiment was unified in the right basal ganglia) and was located $0.2 \mathrm{~mm}$ rostral to and $3.5 \mathrm{~mm}$ lateral to the anterior fontanelle (bregma). After stereotaxic positing, a cranial drill was used to drill a 1-mm-diameter hole through the skull to the dura mater. A $100 \mu \mathrm{L}$ microsyringe (Hamilton Company, Reno, NV) was fixed to a vertical position on the stereotaxic frame, and the needle head and dental drill were then aligned. The position of the stereotactic frame was fixed, and the microsyringe was removed. The hind limbs of the rat were then repositioned to face upward. The abdominal skin was disinfected, and a ventral midline laparotomy incision of $\sim 2-3 \mathrm{~mm}$ in length was made. The caudal artery was carefully separated and exposed under a dissecting microscope. The region was rinsed with normal saline, and the caudal artery was punctured with a microsyringe needle to collect $100 \mu \mathrm{L}$ of arterial blood without anticoagulant. After repositioning the animal in the stereotaxic frame, the microsyringe needle was slowly and vertically inserted through the dental drill until it reached a depth of $\sim 5.5 \mathrm{~mm}$. Next, $100 \mu \mathrm{L}$ of autologous arterial blood was injected into the basal ganglia at a rate of $20 \mu \mathrm{L} / \mathrm{min}$. After the injection, the needle was retained for $10 \mathrm{~min}$ to prevent bleeding. The microsyringe needle was slowly withdrawn, and sterilized bone wax was used to seal the hole in the skull. After confirming that there was no 
hemorrhage, the incision and head skin were sutured, and the tail incision was bandaged with pressure. Each animal received a subcutaneous injection of $5 \mathrm{ml}$ normal saline for postoperative fluid replacement to prevent excessive loss of body fluid. Heart rate, blood pressure, and real-time body and rectal temperature were monitored for approximately $2 \mathrm{~h}$, and temperature was maintained at $37.5{ }^{\circ} \mathrm{C}$, until recovery from anesthesia. According to the four-level evaluation of neurological symptoms described by Bederson et al. [25], rats with neurological symptoms up to one to three level(s) were considered successfully established ICH models. Brain tissues were sampled $1 \mathrm{~mm}$ away from the hematoma to avoid potential red blood cell contamination for further study (Fig. 1a).

\section{Intracerebroventricular injection of pDNA or siRNA}

The drilling site of the intracerebroventricular region for each rat was determined as described in previous studies $[26,27]$. The relevant dosage of pDNA or small interfering RNA (siRNA) for intracerebroventricular injection was in accordance with manufacturer instructions. The drilling site was located $0.2 \mathrm{~mm}$ caudal to and $1.6 \mathrm{~mm}$ lateral to the anterior fontanelle (bregma). The depth of the drilling site was $4.5 \mathrm{~mm}$.

\section{Microglia culture}

Primary microglia-enriched cultures were prepared from the whole brains of 1-day-old pups as described previously [28]. Rats were fixed on a dissection platform and disinfected using $75 \%$ alcohol. The skin and skull were opened, and brain tissues were collected. Brain tissues were washed once in PBS and isolated from the brain stem. The meninges and blood vessels were removed, and the brain tissues were placed in serum-free DMEM/ F12 culture medium. The tissues were cut into small pieces and transferred to centrifuge tubes. Next, $0.125 \%$ trypsin was added, and the brain tissue was digested in a water bath at $37^{\circ} \mathrm{C}$ for $15 \mathrm{~min}$. Trypsinization was terminated by adding a serum solution. After the tissue was precipitated, the supernatant was collected, and the remaining tissue was homogenized and trypsinized before filtering the tissue lysate. The filtered solution was centrifuged at $1500 \mathrm{rpm}$ for $5 \mathrm{~min}$. The supernatant was removed, and the cells were resuspended in complete culture medium before cell counting. The culture medium was changed every 2 days, and stratified cell layers were observed $\sim 10$ days later. Cells in the upper layer, which were mainly microglia, and a small number of oligodendrocytes, were semi-adherent and round with
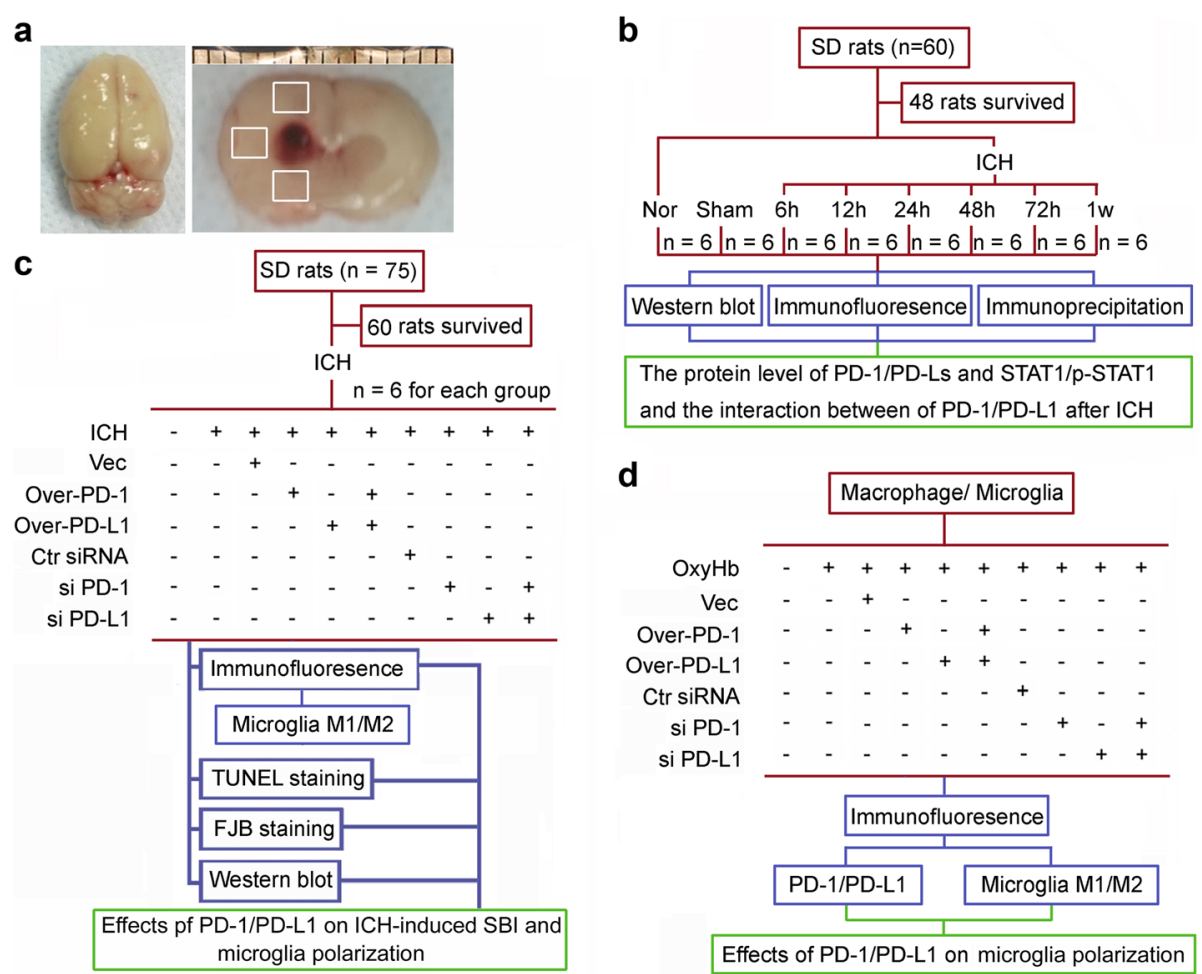

Fig. 1 Flow chart showing rat brain tissue after ICH modeling and experimental design. a Left panel: representative image of completed isolation of rat brain tissue after cardiac perfusion. Right panel: brain section from a rat after ICH modeling. The boxed areas represent the brain regions used for western blot analysis and immunofluorescence staining. b, c Experimental design for in vivo experiment. $\mathbf{d}$ Experimental design for in vitro experiment 
good light transmission. Cells in the lower layer were mainly astrocytes and neurons. Subsequently, culture flasks covered with glial cells/neurons were placed on a shaker and mixed at $180 \mathrm{rpm}$ for $15 \mathrm{~min}$. The culture medium was then collected, non-adherent cells were washed once with PBS, and the cells were centrifuged at $1500 \mathrm{rpm}$ for $5 \mathrm{~min}$. After removing the supernatant, cell pellets were resuspended with complete culture medium and incubated with $5 \% \mathrm{CO}_{2}$ at $37{ }^{\circ} \mathrm{C}$ for $15-$ $30 \mathrm{~min}$. After changing the culture medium, the adhered cells in the culture flasks were purified microglia.

\section{Experimental groups}

To examine PD-1 and PD-Ls expression at different time points after $\mathrm{ICH}$, rats were divided into three groups: normal control group $(n=6)$, sham group $(n=6)$, and $\mathrm{ICH}$ group $(n=36)$ (Fig. 1b). Normal control group animals were not subjected to a procedure. Sham group animals underwent withdrawal of tail artery blood and sphenotresia via microsyringe needle puncture reaching basal ganglia but not blood vessels. ICH group animals were subjected to successful ICH modeling. The ICH group was randomly subdivided into six groups ( $n=6$ each) to collect brain samples at the indicated time points: postICH $6 \mathrm{~h}$, post-ICH $12 \mathrm{~h}$, post-ICH $24 \mathrm{~h}$, post-ICH $48 \mathrm{~h}$, post-ICH $72 \mathrm{~h}$, and post-ICH $7 \mathrm{~d}$. Rat brain tissues in the sham group were collected $48 \mathrm{~h}$ after sphenotresia.

To assess the effect of PD-1/PD-L1 on brain injury, rats were grouped as follows $24 \mathrm{~h}$ after ICH modeling (Fig. 1c). Seventy-five rats were randomly divided into ten groups: sham group $(n=6)$; ICH group $(n=6)$; vector group ( $n=6 / 7$, number of successful ICH models/ total number of ICH models, same below); ICH + PD-1 overexpression group $(n=6 / 7)$; ICH + PD-L1 overexpression group $(n=6 / 9), \mathrm{ICH}+\mathrm{PD}-1$ overexpression + PD-L1 overexpression group $(n=6 / 7) ; \mathrm{ICH}+$ control siRNA group ( $n=6 / 7)$; $\mathrm{ICH}+\mathrm{PD}-1$ siRNA group $(n=6 / 8) ; \mathrm{ICH}$ + PD-L1 siRNA group $(n=6 / 8)$; and ICH + PD-1 siRNA + PD-L1 siRNA $(n=6 / 10)$. pDNA or siRNA was intracerebroventricularly injected into the rats $12 \mathrm{~h}$ before modeling. Animals were killed to collect brain tissue $24 \mathrm{~h}$ after ICH onsets. The serum was collected for enzyme-linked immunosorbent assay (ELISA). DNA and siRNA transfection efficiencies of each sample were verified using western blot analysis. Subsequently, effects of PD-1/PD-L1 on SBI and microglia polarization after ICH were determined using terminal dexynucleotidyl transferase(TdT)-mediated dUTP nick end labeling (TUNEL) staining, Fluoro-Jade B (FJB) staining, western blot analysis, and immunofluorescence staining.

To examine the effect of PD-1/PD-L1 on microglia polarization in vitro (Fig. 1d), cultured microglia were randomly divided into ten groups: control group, $\mathrm{OxyHb}$ group, $\mathrm{OxyHb}+$ vector group, $\mathrm{OxyHb}+\mathrm{PD}-1$ overexpression group, OxyHb + PD-L1 overexpression group, $\mathrm{OxyHb}+\mathrm{PD}-1$ overexpression + PD-L1 overexpression group, $\mathrm{OxyHb}+$ control siRNA group, $\mathrm{OxyHb}+$ PD-1 siRNA group, OxyHb + PD-L1 siRNA group, and $\mathrm{OxyHb}+\mathrm{PD}-1$ siRNA + PD-L1 siRNA group. pDNA or siRNA was transfected to the cultured microglia populations, and after $24 \mathrm{~h}$, the cells were fixed with $4 \%$ formaldehyde. pDNA and siRNA transfection efficiencies of each sample were verified using western blot analysis, followed by microglia polarization analysis.

\section{pDNA and siRNA for PD-1/PD-L1 and transfection}

Specific pDNA and siRNA for PD-1 and PD-L1 were obtained from Guangzhou Ribo Biotechnology Co., Ltd. (Guangzhou, China). To improve the knockdown efficiency, the interference efficiency of three different siRNAs (shown below) was test, and the most efficient one (I for PD-1, III for PD-L1) was used in the following study.

PD-1 siRNA sequences:

(I) Sense: $5^{\prime}$ CCACCUUCACCUGCAGUUU dTdT 3' Antisense: $3^{\prime}$ dTdT GGUGGAAGUGGACGUC AAA $5^{\prime}$

(II) Sense: 5' CCGCUUCCAGAUCGUACAA dTdT 3' Antisense: $3^{\prime}$ dTdT GGCGAAGGUCUAGCAU GUU 5'

(III)Sense: $5^{\prime}$ CCUUCUGCUCAACAGGUAU dTdT 3' Antisense: $3^{\prime}$ dTdT GGAAGACGAGUUGUCC AUA $5^{\prime}$

PD-L1 siRNA sequences:

(I) Sense: 5' GCAGAUUCCCAGUAGAACA dTdT 3' Antisense: $3^{\prime}$ dTdT CGUCUAAGGGUCAUCU UGU $5^{\prime}$

(II) Sense: $5^{\prime}$ GCCGAAGUGAUCUGGACAA dTdT 3' Antisense: $3^{\prime}$ dTdT CGGCUUCACUAGACCU GUU 5'

(III)Sense: 5' GGUCAACGCAACAGCUAAU dTdT 3' Antisense: $3^{\prime}$ dTdT CCAGUUGCGUUGUCGA UUA $5^{\prime}$

pDNA and siRNA sequences were dissolved in RNasefree water to a concentration of $500 \mathrm{pmol} / 10 \mu \mathrm{L}$ and then diluted with the same volume of transfection reagent. For each rat, $4 \mathrm{~g}$ of nucleic acid was injected intracerebroventricularly.

\section{TUNEL staining}

Paraffin-embedded brain sections (4-6 $\mu$ m thick) were incubated at $70{ }^{\circ} \mathrm{C}$ for $1 \mathrm{~h}$, followed by dewaxing and rehydrating in xylene and graded ethanol $(100,95,90,80$, and $70 \%)$ solutions. TUNEL fluorescence staining reagents (Roche) were used in accordance with the manufacturer's 
instructions. Anti-quenching mounting medium was used to seal tissue sections between glass slides and cover slips. Brain sections were observed under a fluorescence microscope, and Image J software was used to analyze TUNEL staining. Six microscopic fields in each tissue section and three sections per rat were examined and photographed in parallel for TUNEL-positive cell counting. Microscopy was performed by an experienced pathologist blind to the experimental condition. The number of TUNEL-positive cells was assessed in $\geq 300$ cells.

\section{FJB staining}

After dewaxing the paraffin-embedded brain sections (described above), the samples were placed in the dark at room temperature and incubated with $0.06 \% \mathrm{KMnO}_{4}$ solution for $15 \mathrm{~min}$. Sections were then washed three times with PBS (5 min/wash), incubated with FJB working solution (containing $0.1 \%$ acetic acid solvent) for $60 \mathrm{~min}$., and washed three times with PBS (5 min/wash). Brain sections on glass slides were air-dried at room temperature in a dark room and sealed with anti-quenching mounting medium and cover slips. Fluorescence microscopy was used for observation and photography. Six microscopic fields in each tissue section and three sections per rat were examined and photographed in parallel for FJB-positive cell counting. Microscopy was performed by an experienced pathologist blind to the experimental condition. The number of FJB-positive cells was assessed in $\geq 300$ cells.

\section{Antibodies}

Mouse anti-PD-1 antibody (MA5-15780), rabbit anti-PDL1 antibody (PA5-20343), and rabbit anti-PD-L2 antibody (PA5-20344) were form Thermo Fisher (USA). p-STAT1 antibody (ab29045), STAT1 antibody (ab31369), rabbit anti-CD16 antibody (ab109223), anti-iNOS antibody (ab15323), anti-TNF alpha antibody (ab6671), anti-IL1 beta antibody (ab9722), anti-IL4 antibody (ab9811), and anti-IL10 antibody (ab9969) were from Abcam (Cambridge, MA, USA). Arginase 1 antibody (GTX109242) was form GeneTex (USA). Mouse anti-CD11b antibody (sc-516102), goat anti-CD206 antibody (sc-34577), mouse anti- $\beta$-actin (C4) antibody (sc-47778), normal mouse IgG (sc-2025), normal rabbit IgG (sc-2027), and normal goat IgG (sc-3887) were from Santa Cruz Biotechnology. Secondary antibodies for western blot analysis, including goat anti-rabbit IgG-HRP (sc-2004), goat anti-mouse IgG-HRP (sc-2005), and rabbit anti-goat IgG-HRP (sc-2922) were from Santa Cruz Biotechnology. Secondary antibodies for immunofluorescence microscopy, including Alexa Fluor-555 donkey anti-rabbit IgG antibody (A31572), Alexa Fluor-488 donkey anti-rabbit IgG antibody (A21206), Alexa Fluor-555 donkey anti- mouse IgG antibody (A31570), Alexa Fluor-488 donkey anti-goat IgG antibody (A11055), Alexa Fluor-488 goat anti-mouse IgG antibody (A-11001), Alexa Fluor555 donkey anti-goat IgG antibody (A-21432), and Alexa Fluor-647 goat anti-rabbit IgG antibody (A32733) were from Invitrogen.

\section{Immunohistochemical study}

The brain samples were fixed in $4 \%$ paraformaldehyde, embedded in paraffin, cut into $4-\mu \mathrm{m}$ sections, and examined by immunofluorescence staining. Then, the sections were stained with primary antibodies (all diluted 1:200; from Santa Cruz Biotechnology, Inc.) and appropriate secondary antibodies (1:500 dilution; Santa Cruz Biotechnology, Inc.) as described. Normal rabbit IgG was used as a negative control for immunofluorescence assay (data not shown). Finally, sections were observed by a fluorescence microscope (OLYMPUS BX50/BX-FLA/ DP70; Olympus Co., Japan). For fluorescence intensity assay, the relative fluorescence intensity was analyzed by use of Image J program by subtracting background. For positive cell counting, six microscopic fields in each tissue section and three sections per rat were examined and photographed in parallel for positive cell counting. Microscopy was performed by an experienced pathologist blind to the experimental condition. The number of positive cells was assessed in $\geq 100$ cells.

\section{Western blot analysis}

The brain tissue around hematoma was thoroughly ground on ice. RIPA lysis buffer (Beyotime Biotechnology) was used to lyse the brain tissue for $30 \mathrm{~min}$. The lysate was then transferred to a centrifuge tube and centrifuged at $12,000 \mathrm{rpm}$ for $5 \mathrm{~min}$ at $4{ }^{\circ} \mathrm{C}$. The supernatant from each sample was collected and stored at $-20{ }^{\circ} \mathrm{C}$. Total protein extracted from each sample was quantified using a BSA protein concentration assay kit (Beyotime Biotechnology). Fifty micrograms of total protein from each sample was separated using SDSPAGE. After transferring the protein gel to a PVDF membrane, corresponding proteins were probed with different antibodies and signals were detected using an ECL kit. ImageJ software was used to analyze optical density of bands.

\section{ELISA}

The concentrations of IL- $1 \beta$ and TNF- $\alpha$ in serum were determined by corresponding ELISA kits (R\&D Systems Inc., USA). These assays were performed according to manufacturer's instructions, and these data were expressed relative to standard curves prepared for them. 


\section{Co-immunoprecipitation (Co-IP)}

Protein A + G agarose for IP was washed twice with lysis buffer. Total protein $(100 \mu \mathrm{g})$ from each sample was diluted in $500 \mu \mathrm{L}$ lysis buffer. Approximately $1 \mu \mathrm{g}$ of conventional IgG, which was identical to the IgG used for IP, was added with $25 \mu \mathrm{L}$ fully resuspended protein $\mathrm{A}+$ $\mathrm{G}$ agarose and gently mixed on a rocker at $4{ }^{\circ} \mathrm{C}$ for $30 \mathrm{~min}$. Samples were then centrifuged at $1000 \times g$ for $5 \mathrm{~min}$ at $4{ }^{\circ} \mathrm{C}$, and the supernatant was collected for subsequent IP and removal of nonspecific antigens. Next, protein solution was added with $2 \mu \mathrm{g}$ of the corresponding primary antibody, and the mixture was incubated on a rocker at $4{ }^{\circ} \mathrm{C}$ for $1 \mathrm{~h}$. After thorough binding between antigen and primary antibody, $50 \mu \mathrm{L}$ of fully resuspended protein $\mathrm{A}+$ $G$ agarose was added, and the reaction was gently mixed on a rocker at $4{ }^{\circ} \mathrm{C}$ overnight. The mixture was then centrifuged at $1000 \times g$ for $5 \mathrm{~min}$ at $4{ }^{\circ} \mathrm{C}$, and the supernatant was discarded. The beads were washed three times with lysis buffer, and $75 \mu \mathrm{L} 4 \times$ SDS sample buffer was added. The sample was boiled at $100{ }^{\circ} \mathrm{C}$ for $5 \mathrm{~min}$ and then centrifuged at $1000 \times g$ for $5 \mathrm{~min}$ at $4{ }^{\circ} \mathrm{C}$. The supernatant was collected and placed in a new Eppendorf tube. Each prepared sample was used directly in western blot analysis.

\section{Data analysis}

All data are expressed as mean \pm SEM. One-way ANOVA for multiple comparisons and Student-Newman-Keuls post hoc test were performed for TUNEL and FJB staining, immunofluorescence staining, immunoprecipitation analysis, and western blot. $p<0.05$ was considered statistically significant.

\section{Results}

$\mathrm{ICH}$-induced increase in the expressions of PD-1 and PD-L1 and the interaction between them in the brain tissue around hematoma in rats

Expression of PD-1 protein in brain tissues from $\mathrm{ICH}$ model rats significantly increased at $24 \mathrm{~h}$ after $\mathrm{ICH}$ onsets. Results of western blot analysis showed that PD-1 protein expression in post-ICH $24 \mathrm{~h}$, post-ICH $48 \mathrm{~h}$, and post-ICH $72 \mathrm{~h}$ subgroups of $\mathrm{ICH}$ rats were significantly higher than that in the sham group (Fig. 2a). In addition, PD-L1 protein expression in post-ICH $24 \mathrm{~h}$ and post- $\mathrm{ICH} 48 \mathrm{~h}$ subgroups of $\mathrm{ICH}$ rats also increased. This enhanced expression of PD-L1 protein decreased in the post-ICH $72 \mathrm{~h}$ subgroup, which had similar PD-L1 protein expression levels as the control group (Fig. 2a). No significant changes in PD-L2 protein expression were found in the different groups (Fig. 2a). In addition, $\mathrm{ICH}$ also increased the interaction between PD-1 and PD-L1 at post-ICH $12 \mathrm{~h}$, post-ICH $24 \mathrm{~h}$, and post-ICH $48 \mathrm{~h}$ (Fig. 1b, c). And the protein levels of PD-1 and PD-L1 and the interaction between them, as well as the phosphorylation of STAT1, collectively peaked at $24 \mathrm{~h}$ after ICH. Therefore, we selected the post-ICH $24 \mathrm{~h}$ subgroup to study the effect of PD1/PD-L1 on ICH-induced SBI.
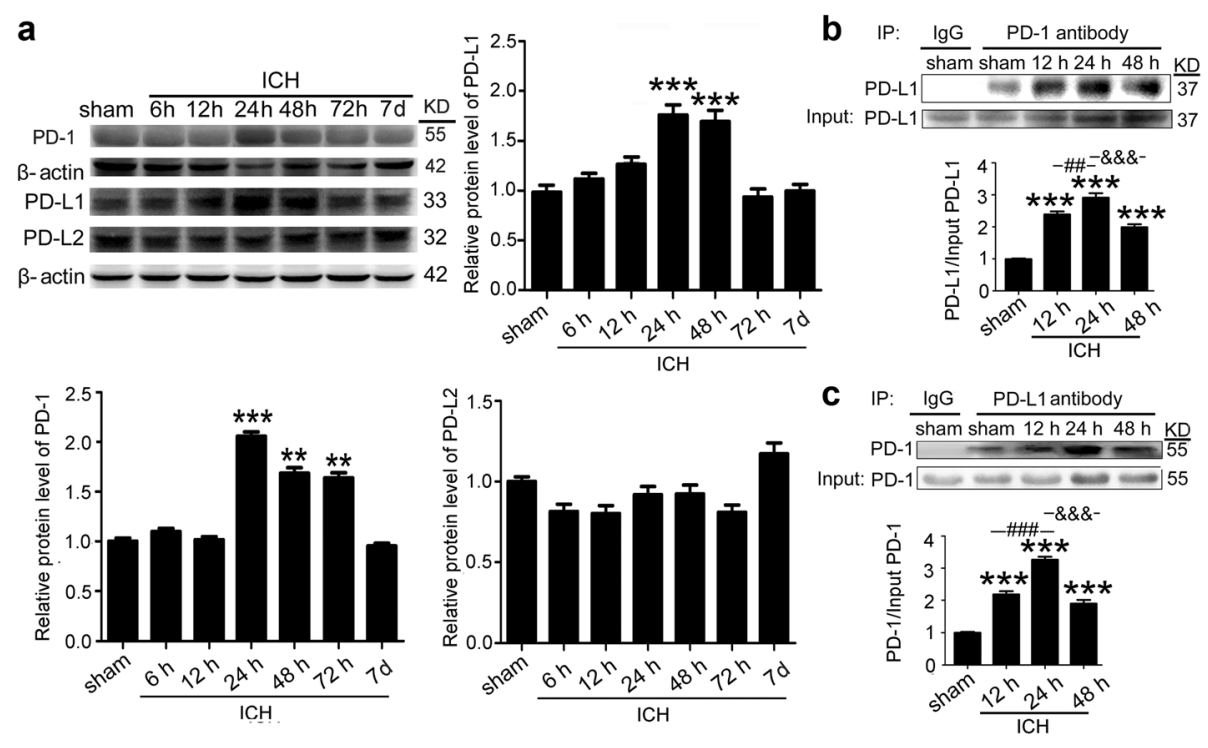

Fig. 2 ICH increased the protein levels of PD-1/PD-Ls and the interaction between PD-1 and PD-L1. a Time course of the protein levels of PD-1, PD-L1, and PD-L2 in the brain tissue around hematoma after ICH. Representative western blot bands of PD-1, PD-L1, and PD-L2 and quantitative analysis of the relative protein level were shown. The mean value of sham group was normalized to 1.0. Data are expressed as mean $\pm S E M, n=6$. Double asterisks indicate $p<0.01$, triple asterisks indicate $p<0.001$ vs. sham group. $\mathbf{b}, \mathbf{c}$ Immunoprecipitation analysis of the interaction between PD-1 and PD-L1 at indicated times after ICH. All values are means \pm SEM, $n=6$. triple asterisks indicate $p<0.001$ vs. sham group, triple pound signs indicate $p<0.001$ 
Effects of overexpression and knockdown of PD-1 and PD-L1 on brain cell death and neuronal degeneration in $\mathrm{ICH}$ rats

The experimental group of Fig. 3 is shown in Fig. 3a. Western blot assay showed that the protein levels of PD-1 and PD-L1 were significantly increased by pDNA transfection and decreased by siRNA transfection (Fig. 3b, c). TUNEL assay and FJB staining were used to assess the effects of PD-1/PD-L1 on brain cell death and neuronal degeneration in the brain after ICH modeling (Fig. 3d-g). The numbers of TUNEL- and FJB-positive cells in rat brains $24 \mathrm{~h}$ after ICH modeling were significantly higher than those in the sham group. However, overexpression of both PD-1 and PD-L1 significantly inhibited ICH-induced brain cell death and neuronal degeneration, while knockdown of them exerted an opposite effect. In addition, inflammation-associated molecules, including IL-1 $\beta$ and TNF- $\alpha$, were tested. As shown in Fig. $3 \mathrm{~h}, \mathrm{i}, \mathrm{IL}-1 \beta$ and TNF- $\alpha$ were found to be significantly higher in the serum of the ICH group than that in the sham group. Compared with $\mathrm{ICH}$ group, the mean inflammatory cytokine contents were lower in overexpression group and higher in knockdown group. The results also showed that there were no significant differences between the vector group and PD-1 plasmid group or PD-L1 plasmid group, while there were significant differences between the control siRNA group and PD-1 siRNA group or PD-L1 siRNA group, suggesting that PD-1 and PD-L1complement each other in modulation of brain cell death and neuronal degeneration in $\mathrm{ICH}$ rats.

\section{Effects of PD-1 and PD-L1 on ICH-induced microglia polarization and STAT1 phosphorylation in rats}

We next observed the effects of PD-1 and PD-L1 overexpression or knockdown on the microglia polarization in $\mathrm{ICH}$ rat brain (Fig. 4). The experimental group of Fig. 4 is shown in Fig. 4a. The results showed that $\mathrm{ICH}$ induced microglia polarization to a pro-inflammatory phenotype, as defined by CD16/CD11b-positive, and also to an anti-inflammatory phenotype, as defined by CD206/CD11b-positive (Fig. 4b-e). The results also showed that ICH mainly selectively modulated microglia polarizing to pro-inflammatory phenotype, which was ameliorated by overexpression of PD-1 and PD-L1 and aggravated by knockdown of PD-1 and PD-L1. To further elucidate the role of PD-1 and PD-L1 in microglia polarization, we co-stained brain sections of $\mathrm{ICH}+\mathrm{PD}-1$ overexpression + PD-L1 overexpression group for PD1, CD16 and CD11b or PD1, CD206, and CD11b (Fig. 4f). The results demonstrated that almost all of the CD206/ CD11b-positive cells were co-labeled with high fluorescence intensity of PD1, while almost all of the CD16/ CD11b-positive cells showed almost undetectable fluorescence intensity of PD1.
In addition, we used western blot analysis to measure the phosphorylation level of STAT1. Our results indicated that the phosphorylation level of p-STAT1 after ICH modeling was significantly higher than that in the sham group and peaked at $24 \mathrm{~h}$ after ICH onsets (Fig. 4g). And the phosphorylation level of STAT1 was decreased by overexpression of PD-1 and PD-L1 and further increased by knockdown of PD-1 and PD-L1 (Fig. 4h).

\section{Critical role of PD-1 and PD-L1 in microglia polarization in cultured microglia under $\mathrm{OxyHb}$ treatment}

We next observed the effects of PD-1 and PD-L1 overexpression or knockdown on microglia polarization in cultured microglia under OxyHb treatment (Fig. 5). The experimental group of Fig. 5 is shown in Fig. $5 \mathrm{a}$. Firstly, the expression plasmid-mediated overexpression of PD1 and PD-L1 as well as the efficiency of siRNAmediated knockdown in cultured microglia is verified by immunofluorescence staining (Fig. 5b). In addition, double-immunofluorescence analysis showed that $\mathrm{OxyHb}$ treatment induced microglia polarization both to a proinflammatory phenotype and an anti-inflammatory phenotype (Fig. $5 \mathrm{c}-\mathrm{f}$ ). The results also showed that $\mathrm{OxyHb}$ treatment mainly selectively modulated microglia polarizing to pro-inflammatory phenotype, which was ameliorated by overexpression of PD-1 and PD-L1 and aggravated by knockdown of PD-1 and PD-L1. To further elucidate the role of PD-1 and PD-L1 in microglia polarization, we co-stained brain sections of $\mathrm{OxyHb}+\mathrm{PD}$ 1 overexpression + PD-L1 overexpression group for PD1, CD16 and CD11b or PD1, CD206, and CD11b (Fig. 5g). The results demonstrated that almost all of the CD206/ CD11b-positive cells were co-labeled with high fluorescence intensity of PD1, while almost all of the CD16/ CD11b-positive cells showed almost undetectable fluorescence intensity of PD1.

In addition, as previously reported [29], the use of single phenotypic markers (CD206 and CD16) is not sufficient to identify microglia polarization. Inflammationassociated molecules, including TNF- $\alpha$, IL- $1 \beta$, iNOS, arginase1, IL-4, and IL-10, were tested to provide more information on the biological state of microglia after ICH and the roles of PD-1 and PD-L2 in the process (Fig. 6). The results showed that the mean protein levels of pro-inflammation molecules, including TNF- $\alpha$, IL- $1 \beta$, and iNOS, in cultured microglia were significantly increased by $\mathrm{OxyHb}$ treatment. However, among the anti-inflammation molecules, including arginase1, IL-4 and IL-10, only the protein level of arginase1 was increased after $\mathrm{OxyHb}$ treatment. These results suggested that $\mathrm{OxyHb}$ induced microglia polarization to a pro-inflammation phenotype. In addition, a significant decrease in the mean protein levels of TNF- $\alpha$, IL-1 $\beta$, and iNOS and a significant increase in the mean protein levels 


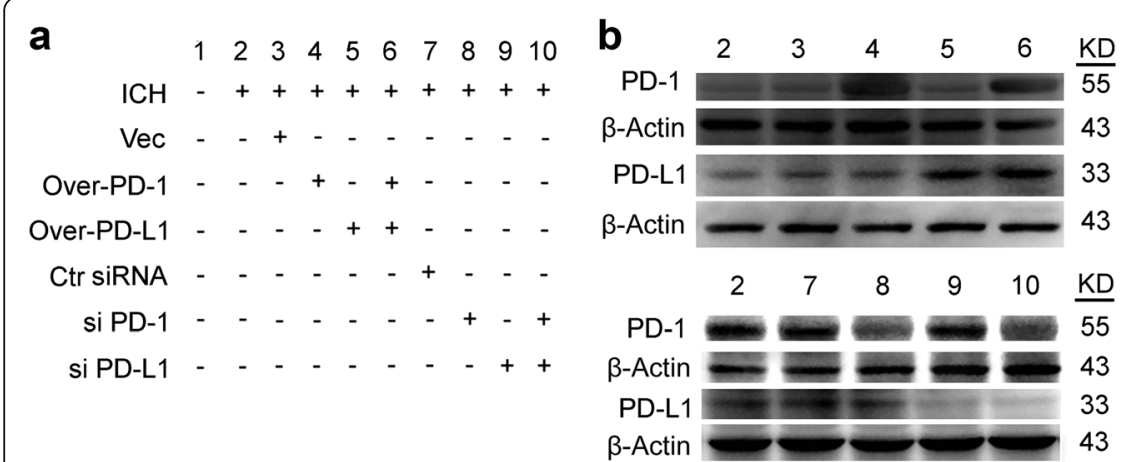

d

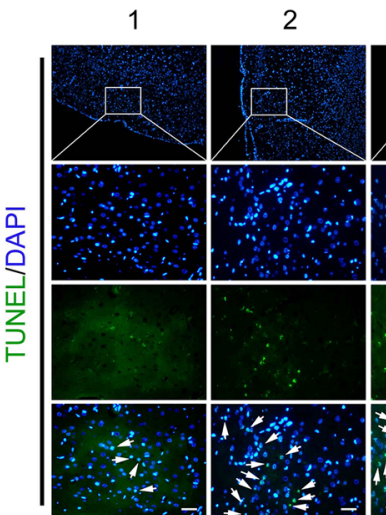
3
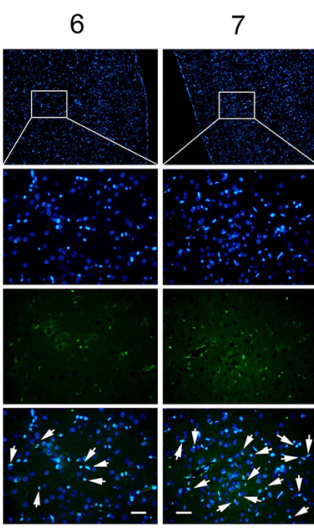

10

f

1

2

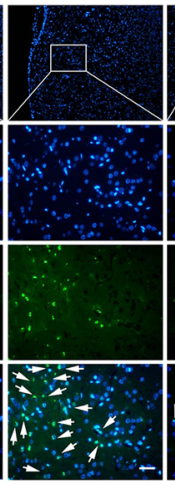

6
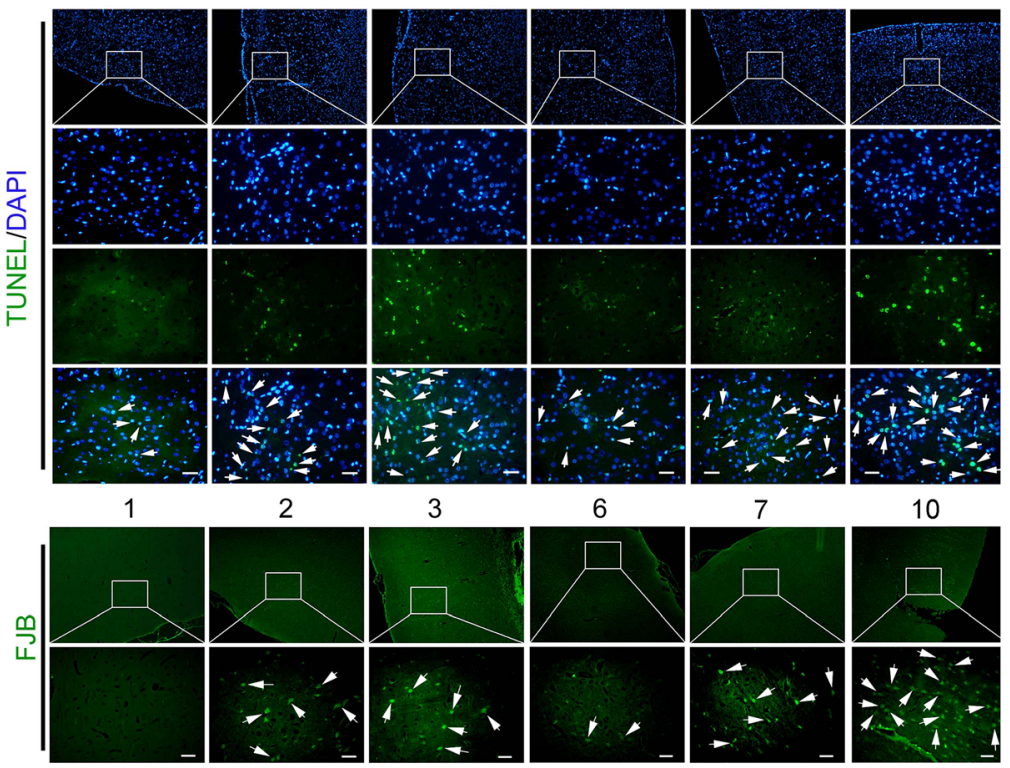

10

e

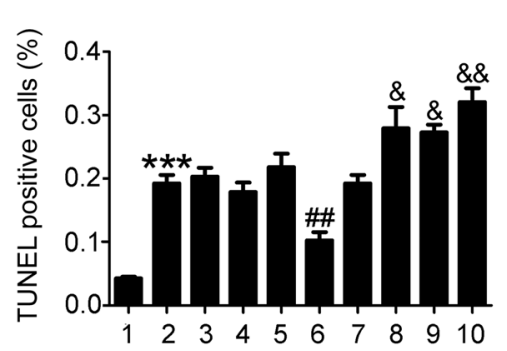

g

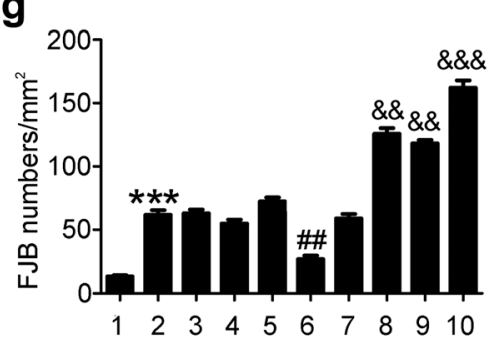

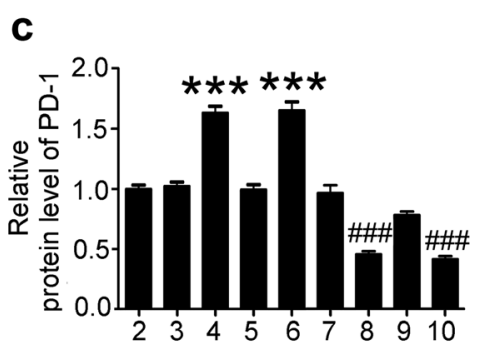

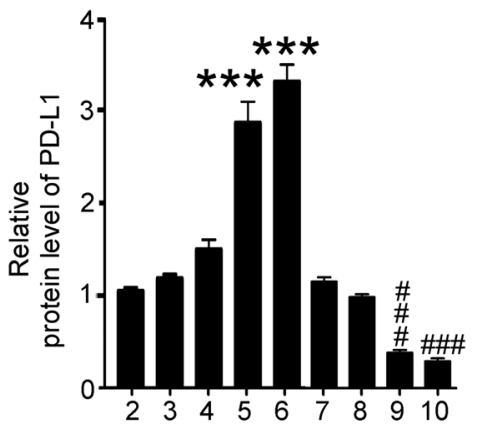

h

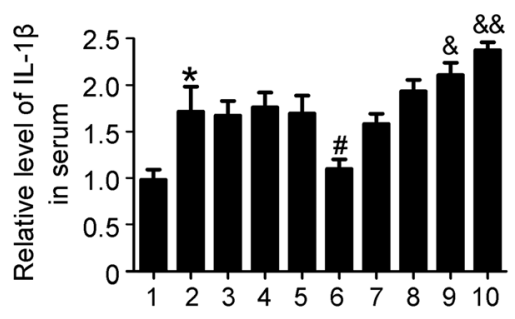

i

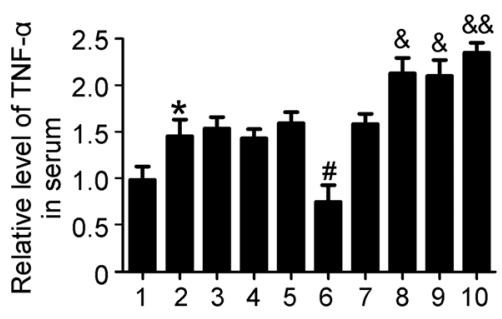

Fig. 3 Effects of PD-1/PD-L1 overexpression and knockdown on brain cell death and neuronal degeneration after ICH. a ICH rats accepted intracerebroventricular injection of pDNA or siRNAs as indicated. $\mathbf{b}$ Western blot analysis of the efficiency of PD-1 and PD-L1 overexpression or knockdown in brain of $\mathrm{ICH}$ rats. Quantification of relative protein levels of PD-1 and PD-L1 was shown in $\mathbf{c}$. $\mathbf{c}$ Data are expressed as mean \pm SEM, $n=6$. Triple asterisks indicate $p<0.001$ vs. ICH + vector group; triple pound signs indicate $p<0.001$ vs. ICH + control siRNA group. $\mathbf{d}$ Terminal deoxynucleotidyl transferase dUTP nick end labeling (TUNEL) staining. Sections were labeled by TUNEL (green) to detect apoptotic brain cells and counterstained with DAPI (b/ue) to detect nuclei. Arrows point to TUNEL-positive cells. Scale bar $=64 \mu \mathrm{m}$. e Percentage of TUNEL-positive cells. Data are expressed as mean \pm SEM, $n=6$. Triple asterisks indicate $p<0.001$ vs. sham group; double pound signs indicate $p<0.01$ vs. ICH + vector group; ampersand indicates $p<0.05$, double ampersands indicate $p<0.01 \mathrm{vs}$. ICH + control siRNA group. f Fluoro-jade B (FJB) staining. Arrows point to FJB-positive cells. Scale bar $=64 \mu \mathrm{m}$. The number of FJB-positive brain cells was calculated. $\mathbf{g}$ Data are expressed as mean $\pm \mathrm{SEM}, n=6$. Triple asterisks indicate $p<0.001$ vs. sham group; double pound signs indicate $p<0.01$ vs. ICH + vector group; double ampersands indicate $p<0.01$, triple ampersands indicate $p<0.001$ vs. ICH + control siRNA group. $\mathbf{h}$, i ELISA assay of the contents of IL-1 $\beta$ and TNF-a in the serum. The mean values of the sham group were normalized to 1.0. Data are expressed as mean \pm SEM, $n=6$. Single asterisk indicates $p<0.05$ vs. sham group; Single pound sign indicates $p<0.05$ vs. ICH + vector group; Single ampersand indicates $p<0.05$, double ampersands indicate $p<0.01$ vs. ICH + control siRNA group 


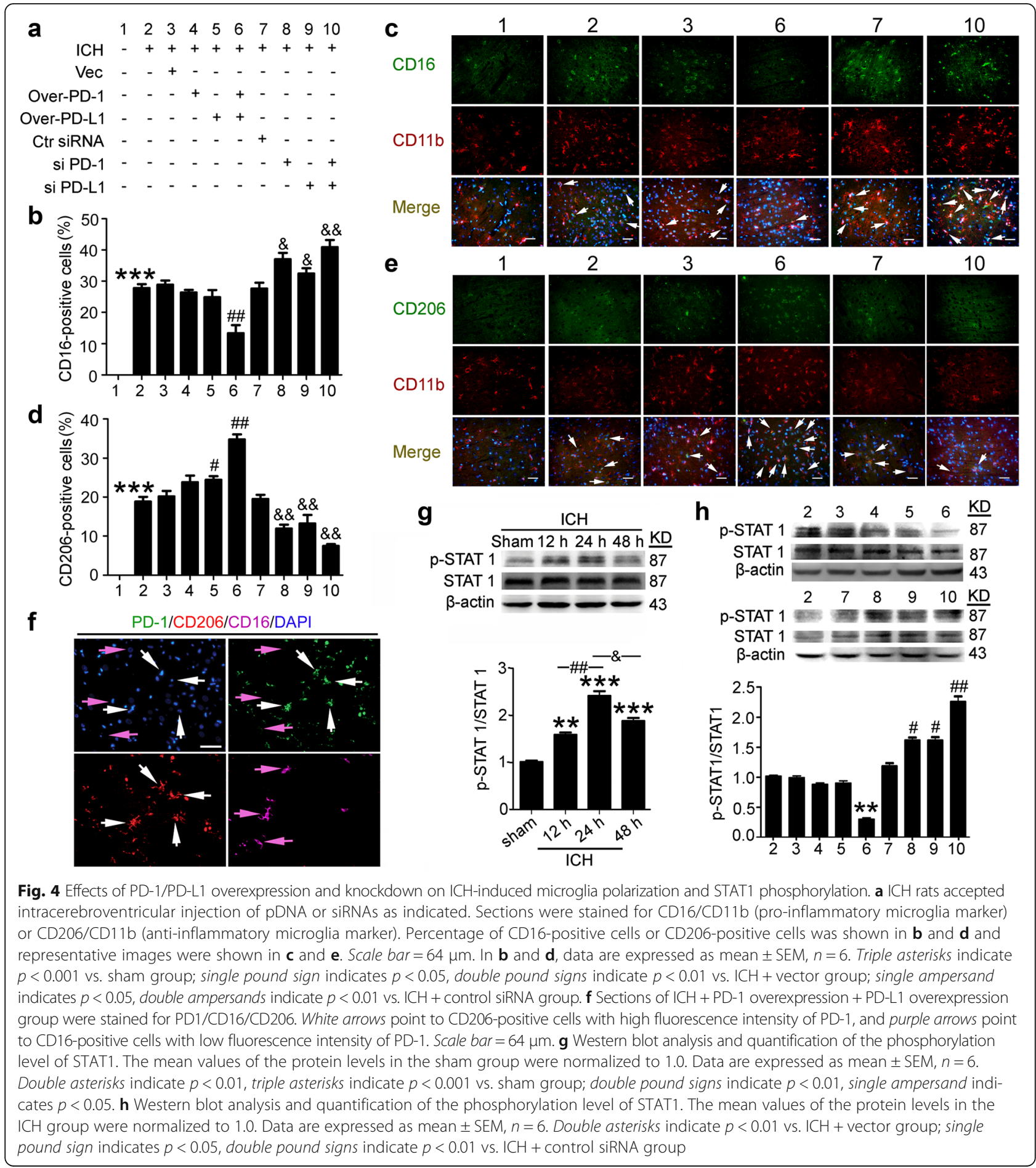

of arginase 1, IL-4, and IL-10 were observed in microglia with PD-1 and PD-L1 overexpression, while opposite trends were shown in microglia with PD-1 and PD-L1 knockdown, suggesting that PD-1 and PD-L1 selectively inhibited $\mathrm{OxyHb}$-induced pro-inflammation polarization of microglia.
In addition, the mean protein levels of proinflammatory markers, including TNF- $\alpha$, IL- $1 \beta$, and iNOS, in cultured microglia were significantly increased by $\mathrm{OxyHb}$ treatment (Fig. 6). However, among the antiinflammatory markers, including arginase 1, IL-4, and IL-10, only the protein level of arginase 1 was increased 


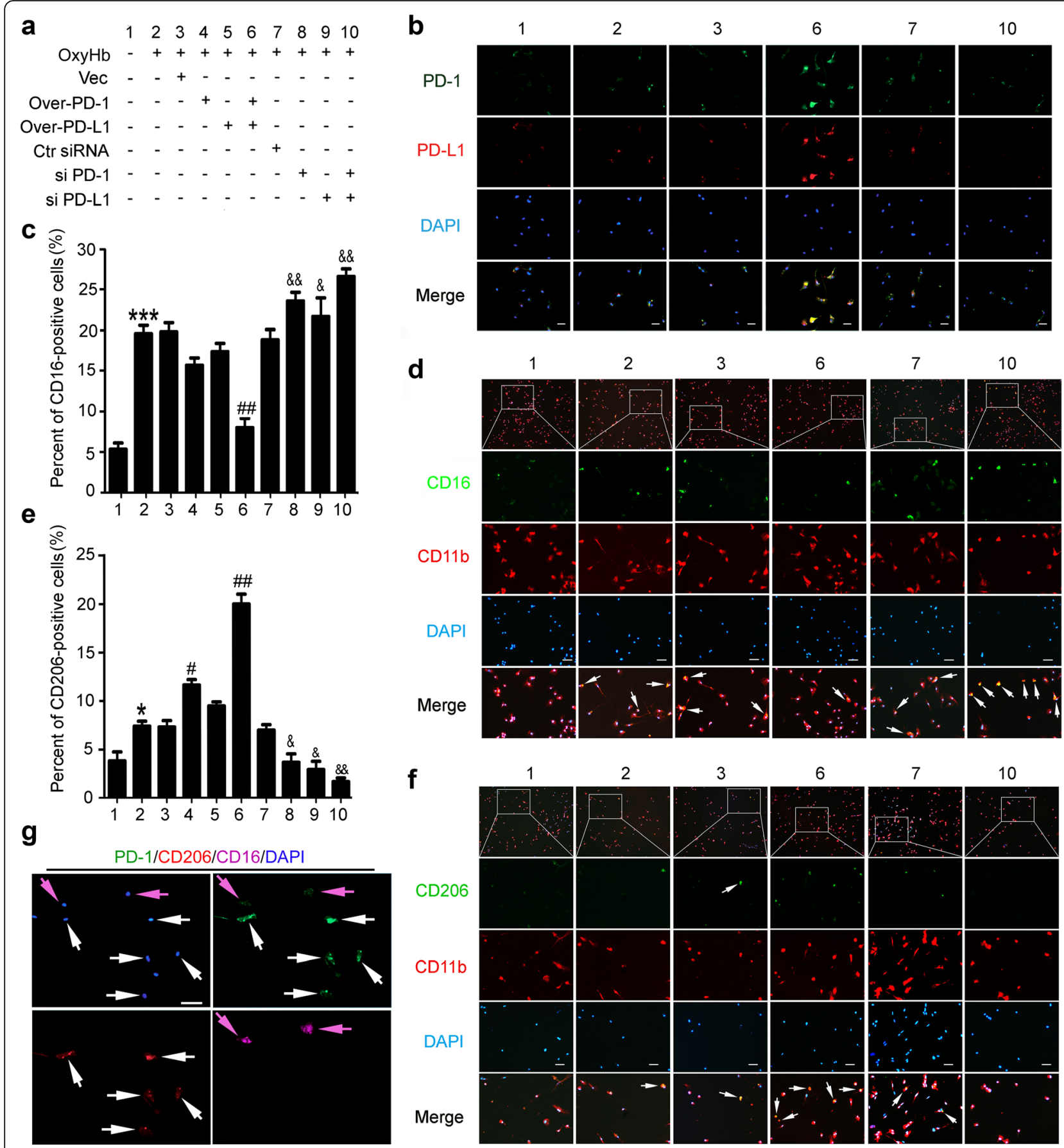

Fig. 5 Effects of PD-1/PD-L1 overexpression and knockdown on the polarization of OxyHb-treated microglia. a Cultured microglia accepted transfection of pDNA or siRNAs as indicated. $\mathbf{b}$ The efficiency of pDNA and siRNA in cultured microglia was verified by immunofluorescence staining. Scale bar $=64 \mu \mathrm{m}$. Cultured microglia was stained for CD16/CD11b (pro-inflammatory microglia marker) or CD206/CD11b (anti-inflammatory microglia marker). Percentage of CD16-positive cells or CD206-positive cells was shown in $\mathbf{c}$ and $\mathbf{e}$ and representative images were shown in $\mathbf{d}$ and $\mathbf{f}$. Scale bar $=64 \mu \mathrm{m}$. In $\mathbf{c}$ and $\mathbf{e}$, data are expressed as mean $\pm \mathrm{SEM}, n=6$. Single asterisk indicates $p<0.05$, triple asterisks indicate $p<0.001 \mathrm{vs}$. sham group; single pound sign indicates $p<0.05$, double pound signs indicate $p<0.01 \mathrm{vs}$. ICH + vector group; single ampersand indicates $p<0.05$, double ampersands indicate $p<0.01$ vs. ICH + control siRNA group. g Microglia of ICH + PD-1 overexpression + PD-L1 overexpression group was stained for PD1/CD16/CD206. White arrows point to CD206-positive cells with high fluorescence intensity of PD-1, and purple arrows point to CD16-positive cells with low fluorescence intensity of PD-1. Scale bar $=64 \mu \mathrm{m}$ 

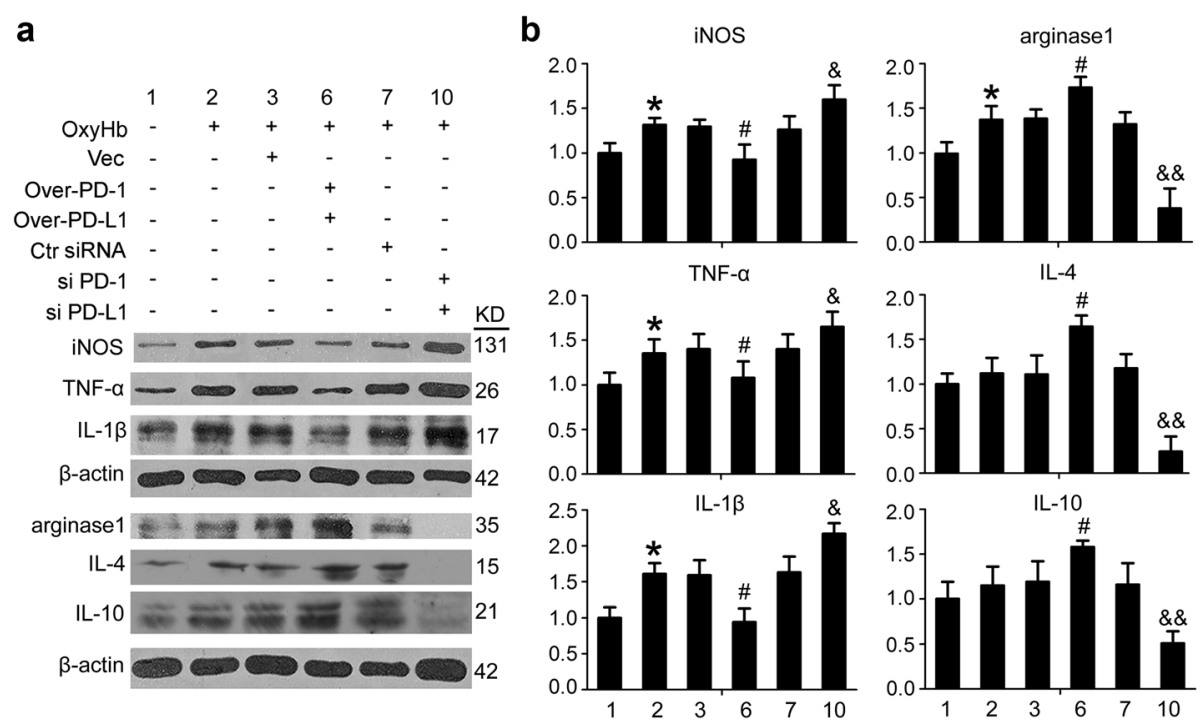

Fig. 6 Changes in the expression of pro-inflammatory- and anti-inflammatory-like polarization markers in microglia under indicated treatment. a The immunoblots show TNF-a, IL-1 $\beta$, iNOS, arginase1, IL-4, and IL-10 produced by microglia under indicated treatment. $\mathbf{b}$ The quantitative analyses of TNF-a, IL-1 $\beta$, iNOS, arginase1, IL-4, and IL-10 in the immunoblots in a. Date $=$ mean $\pm \mathrm{SEM}, n=6$. Single asterisk indicates $p<0.05 \mathrm{vs}$. control group; single pound sign indicates $p<0.05 \mathrm{vs}$. ICH + vector group; single ampersand indicates $p<0.05$, double ampersands indicate $p<0.01 \mathrm{vs}$. ICH + control siRNA group

after OxyHb treatment. These results suggested that OxyHb induced microglia activation and mainly promoted microglia polarization to pro-inflammatory phenotype. A significant decrease in the mean protein levels of pro-inflammatory markers and a significant increase in the mean protein levels of anti-inflammatory markers were observed in microglia with PD-1 and PDL1 overexpression, while opposite trends were shown in microglia with PD-1 and PD-L1 knockdown, suggesting that PD-1 and PD-L1 selectively inhibited OxyHbinduced pro-inflammatory polarization of microglia.

\section{Discussion}

In this study, we established a rat model of ICH to determine whether PD-1/PD-Ls play a role in SBI and to elucidate the potential mechanisms. Our findings showed that PD-1/PD-L1 protein expression in brain tissue significantly increased after ICH. In addition, the proportion of STAT1 phosphorylation increased after ICH. These changes were more significant $\sim 24 \mathrm{~h}$ after ICH. Overexpression of PD1 and PD-L1 inhibited STAT1 phosphorylation, which was an important factor for transforming microglia to the pro-inflammatory subtype. Thus, these results suggested that enhancing the expressions of PD-1 and PD-L1 may induce a selective modulation of microglia polarization to anti-inflammatory phenotype via STAT1 for ICH treatment (Fig. 7). This finding has not been reported in previous studies.

In a study of autoimmune encephalomyelitis, Carter et al. [30] showed that PD-1/PD-L1 but not PD-L2 are involved in $\mathrm{T}$ cell activation. In this study, we demonstrated that PD-1 and PD-L1 but not PD-L2 protein expression significantly reduced early brain injury (Fig. 2). Thus, we suggest that the PD-1/PD-L1 signaling pathway plays an important role in regulating the inflammatory response in nervous tissue. In the ICH rat model, PD-1 and PD-L1 protein expression and their binding to each other were elevated $24 \mathrm{~h}$ after ICH modeling, further indicating

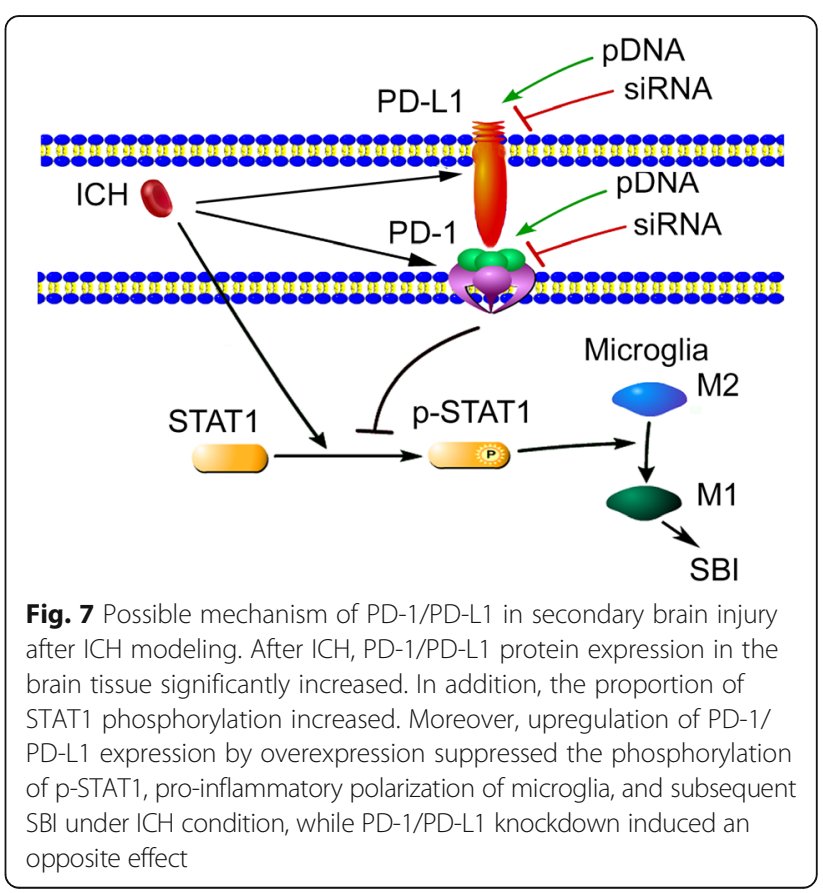


that PD-1/PD-L1 are involved in ICH-induced SBI. The severity of brain injury was alleviated increased protein expression of both PD-1 and PD-L1. In contrast, reduced PD-1 and PD-L1 protein expression increased the severity of brain injury, indicating a causal relationship between PD-1/PD-L1 and brain injury (Fig. 3).

Microglia/macrophages effectively regulate restoration and regeneration of the CNS. However, these molecules can act as double-edged swords, resulting in both destruction and restoration [31]. Further studies have demonstrated that activation of microglia/macrophages can convert the cells into two opposite phenotypes: proinflammatory microglia that release destructive inflammatory cytokines and anti-inflammatory microglia that release cytokines for protection and restoration [25, 32]. In addition, multiple members of the STAT family (i.e., STAT1, STAT3, and STAT6) are involved in morphological typing of microglia/macrophages. For example, STAT1 promotes transformation of microglia into proinflammatory microglia [19, 33], and STAT6 stimulates transformation of microglia into anti-inflammatory microglia [34]. Moreover, STAT1 and STAT6 have a mutually inhibitory relationship [35]. In this study, the ratio of p-STAT1/STAT1 was significantly increased following ICH (Fig. 4g), while ICH did not affect the level of pSTAT6/STAT6 significantly (data not shown). Among the different $\mathrm{ICH}$ rat model groups, the proportion of phosphorylated STAT1 was maximal $24 \mathrm{~h}$ after ICH. PD-1 reduced phosphorylation of STAT1, which was consistent with the result of a previous study [36]. The possible mechanism may be due to PD-1 blocking the PI3K-Akt pathway, which inhibits promotion of phosphorylation of STAT1 [18]. In a mouse model of spinal cord injury, inhibition of PD-1 promotes transformation of microglia/macrophages into pro-inflammatory microglia [36]. These findings support our hypothesis that PD-1/PD-L1 is involved in inhibiting pro-inflammatory polarization of microglia under conditions of ICH [36].

There are several limitations of this study. In vitro experiment showed OxyHb maybe an incentive for the increase in the expressions of PD-1 and PD-L1. However, due to lacking studies of other contents of hematoma, this study cannot draw the mechanism underlying $\mathrm{ICH}$ induced increase in the expressions of PD-1 and PD-L1. In addition, whether ICH-induced increase in the expressions lead to $\mathrm{ICH}$-induced increase in the interaction between PD-1 and PD-L1 is also not answered in this study. It also reported that the PD-L1-mediated inflammatory response expanded the infarct size in experimental stroke model [37]. The possible mechanism may involve binding between PD-L1 and CTLA-4. CTLA-4 inhibits Akt phosphorylation through activation of protein phosphatase 2 (PP2A) [17, 38]. And a study has shown that PD-1 can inhibit PD-L1 protein expression by promoting transcription of MicroRNA 513 [39] to negatively regulate PD-L1 and play a neuroprotective effect in nervous tissue. The effect of CTLA- 4 and MicroRNA 513 on microglia polarization in $\mathrm{ICH}$ condition deserves further study.

\section{Conclusions}

Microglia polarization is part of the basic machinery of brain injury. PD-1/PD-L1, a typical member of immunoglobulin superfamily, plays a critical role in neuroimmune cell functions. On the other hand, much attention has been also paid to the role of STATs in microglia polarization. In this study, using in vivo and in vitro models of relevance to $\mathrm{ICH}$, we found a critical role of PD-1/PD-L1 through STAT1 in the polarization of microglia. These findings suggest PD-1/PD-L1 as promising pharmacological targets for the treatment of hemorrhagic brain injury.

\section{Abbreviations \\ Akt: Protein kinase B; CNS: Central nervous system; Co-P: Co-immunoprecipitation; CTLA-4: Cytotoxic T lymphocytes associated antigen-4; FJB: Fluoro-Jade B ICH: Intracerebral hemorrhage; PD-1: Programmed death protein 1; PD- L1: Programmed death-ligand 1; PI3K: Phosphatidylinositol-3-kinase; SBI: Secondary brain injury; STAT1: Signal transducers and activators of transcription 1; TUNEL: Terminal dexynucleotidyl transferase(TdT)- mediated dUTP nick end labeling \\ Acknowledgements \\ None. \\ Funding \\ This work was supported by Suzhou Key Medical Center (Szzx201501), grants from the National Natural Science Foundation of China (No. 81571115, 81422013, and 81471196), Scientific Department of Jiangsu Province (No. BL2014045), Suzhou Government (No. LCZX201301, SZS201413, and SYS201332), and a project funded by the Priority Academic Program Development of Jiangsu Higher Education Institutions.}

\section{Availability of data and materials}

The authors are willing to make readily reproducible materials, including all relevant raw data, freely available to any scientist wishing to use them. Unfortunately, our institution does not provide a secure mechanism for anonymous sharing. Please email the corresponding authors.

\section{Authors' contributions}

GC and ZY conceived and designed the study, including quality assurance and control. JW and LS performed the experiments and wrote the paper. HL designed the study's analytic strategy. HS helped conduct the literature review and prepare the "Methods" section of the text. WZ reviewed and edited the manuscript. All authors read and approved the manuscript.

\section{Competing interests}

The authors declare that they have no competing interests.

\section{Consent for publication}

Not applicable.

Ethics approval

The animal experimental protocols were approved by the Animal Care and Use Committee of Soochow University and complied with the ARRIVE guidelines. 
Received: 3 June 2016 Accepted: 4 January 2017

\section{Published online: 14 February 2017}

\section{References}

1. Krishnamurthi RV, et al. Global and regional burden of first-ever ischaemic and haemorrhagic stroke during 1990-2010: findings from the Global Burden of Disease Study 2010. Lancet Glob Health. 2013;1(5):e259-81.

2. Behrouz R. Re-exploring tumor necrosis factor alpha as a target for therapy in intracerebral hemorrhage. Transl Stroke Res. 2016;7(2):93-6.

3. Jiang, B., et al., Role of glibenclamide in brain injury after intracerebral hemorrhage. Transl Stroke Res, 2016

4. van Asch CJ, et al. Incidence, case fatality, and functional outcome of intracerebral haemorrhage over time, according to age, sex, and ethnic origin: a systematic review and meta-analysis. Lancet Neurol. 2010;9(2):167-76.

5. Poon MT, Fonville AF, Al-Shahi Salman R. Long-term prognosis after intracerebral haemorrhage: systematic review and meta-analysis. J Neurol Neurosurg Psychiatry. 2014;85(6):660-7.

6. Dang $\mathrm{G}$, et al. Early erythrolysis in the hematoma after experimental intracerebral hemorrhage. Transl Stroke Res. 2016. Pubmed number 27783383. In press.

7. Xi G, Keep RF, Hoff JT. Mechanisms of brain injury after intracerebral haemorrhage. Lancet Neurol. 2006;5(1):53-63.

8. Xiong $X Y$, Yang $Q W$. Rethinking the roles of inflammation in the intracerebral hemorrhage. Transl Stroke Res. 2015;6(5):339-41.

9. Chen $\mathrm{S}$, et al. An update on inflammation in the acute phase of intracerebral hemorrhage. Transl Stroke Res. 2015;6(1):4-8.

10. Venkatesan $\mathrm{C}$, et al. Chronic upregulation of activated microglia immunoreactive for galectin-3/Mac-2 and nerve growth factor following diffuse axonal injury. J Neuroinflammation. 2010;7:32.

11. Loane DJ, Byrnes KR. Role of microglia in neurotrauma. Neurotherapeutics. 2010;7(4):366-77.

12. Amor $\mathrm{S}$, et al. Inflammation in neurodegenerative diseases. Immunology. 2010;129(2):154-69.

13. Jin $\mathrm{X}$, et al. Temporal changes in cell marker expression and cellular infiltration in a controlled cortical impact model in adult male C57BL/6 mice. PLoS One. 2012;7(7):e41892.

14. Wan S, et al. Microglia activation and polarization after intracerebral hemorrhage in mice: the role of protease-activated receptor-1. Transl Stroke Res. 2016;7(6):478-87.

15. Zhao $\mathrm{H}$, et al. Microglia/macrophage polarization after experimental intracerebral hemorrhage. Transl Stroke Res. 2015;6(6):407-9.

16. Ishida $Y$, et al. Induced expression of PD-1, a novel member of the immunoglobulin gene superfamily, upon programmed cell death. EMBO J. 1992;11(11):3887-95.

17. Parry RV, et al. CTLA-4 and PD-1 receptors inhibit T-cell activation by distinct mechanisms. Mol Cell Biol. 2005;25(21):9543-53.

18. Nguyen $\mathrm{H}$, et al. Roles of phosphatidylinositol 3-kinase in interferon-gammadependent phosphorylation of STAT1 on serine 727 and activation of gene expression. J Biol Chem. 2001;276(36):33361-8.

19. Qin $\mathrm{H}$, et al. SOCS3 deficiency promotes M1 macrophage polarization and inflammation. J Immunol. 2012;189(7):3439-48.

20. Butte MJ, et al. Programmed death-1 ligand 1 interacts specifically with the B7-1 costimulatory molecule to inhibit T cell responses. Immunity. 2007;27(1):111-22.

21. Kroner A, et al. A PD-1 polymorphism is associated with disease progression in multiple sclerosis. Ann Neurol. 2005;58(1):50-7.

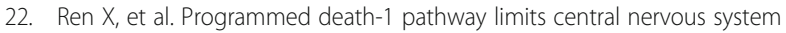
inflammation and neurologic deficits in murine experimental stroke. Stroke. 2011;42(9):2578-83.

23. Saresella M, et al. A potential role for the PD1/PD-L1 pathway in the neuroinflammation of Alzheimer's disease. Neurobiol Aging. 2012;33(3):624 e11-22.

24. Deinsberger $W$, et al. Experimental intracerebral hemorrhage: description of a double injection model in rats. Neurol Res. 1996;18(5):475-7.

25. David S, Kroner A. Repertoire of microglial and macrophage responses after spinal cord injury. Nat Rev Neurosci. 2011;12(7):388-99.

26. Gao C, et al. Role of red blood cell lysis and iron in hydrocephalus after intraventricular hemorrhage. J Cereb Blood Flow Metab. 2014;34(6):1070-5.

27. Gao F, et al. Hydrocephalus after intraventricular hemorrhage: the role of thrombin. J Cereb Blood Flow Metab. 2014;34(3):489-94.

28. Hu X, et al. Macrophage antigen complex-1 mediates reactive microgliosis and progressive dopaminergic neurodegeneration in the MPTP model of Parkinson's disease. J Immunol. 2008;181(10):7194-204.
29. Murray PJ, et al. Macrophage activation and polarization: nomenclature and experimental guidelines. Immunity. 2014;41(1):14-20.

30. Carter $L L$, et al. PD-1/PD-L1, but not PD-1/PD-L2, interactions regulate the severity of experimental autoimmune encephalomyelitis. J Neuroimmunol. 2007;182(1-2):124-34.

31. Hanisch UK, Kettenmann H. Microglia: active sensor and versatile effector cells in the normal and pathologic brain. Nat Neurosci. 2007;10(11):1387-94.

32. Mosser DM, Edwards JP. Exploring the full spectrum of macrophage activation. Nat Rev Immunol. 2008;8(12):958-69.

33. Sica A, Bronte V. Altered macrophage differentiation and immune dysfunction in tumor development. J Clin Invest. 2007;117(5):1155-66.

34. Sheldon KE, et al. Shaping the murine macrophage phenotype: IL-4 and cyclic AMP synergistically activate the arginase I promoter. J Immunol. 2013; 191(5):2290-8

35. Ohmori Y, Hamilton TA. Interleukin-4/STAT6 represses STAT1 and NF-kappa B-dependent transcription through distinct mechanisms. J Biol Chem. 2000; 275(48):38095-103.

36. Yao A, et al. Programmed death 1 deficiency induces the polarization of macrophages/microglia to the M1 phenotype after spinal cord injury in mice. Neurotherapeutics. 2014;11(3):636-50.

37. Bodhankar S, et al. PD-L1 enhances CNS inflammation and infarct volume following experimental stroke in mice in opposition to PD-1. J Neuroinflammation. 2013;10:111.

38. Riley JL. PD-1 signaling in primary T cells. Immunol Rev. 2009;229(1):114-25.

39. Gong AY, et al. MicroRNA-513 regulates B7-H1 translation and is involved in IFN-gamma-induced B7-H1 expression in cholangiocytes. J Immunol. 2009; 182(3):1325-33.

\section{Submit your next manuscript to BioMed Central and we will help you at every step:}

- We accept pre-submission inquiries

- Our selector tool helps you to find the most relevant journal

- We provide round the clock customer support

- Convenient online submission

- Thorough peer review

- Inclusion in PubMed and all major indexing services

- Maximum visibility for your research

Submit your manuscript at www.biomedcentral.com/submit
Biomed Central 